"This document is the Accepted Manuscript version of a Published Work that appeared in final form in The Journal of Physical Chemistry A, copyright $(\mathcal{C}$ American Chemical Society after peer review and technical editing by the publisher. To access the final edited and published work see https://pubs-acs-org.uml.idm.oclc.org/doi/10.1021/acs.jpca.8b11877”.

\title{
Rotational Spectra and Structures of Phenyl Isocyanate and Phenyl Isothiocyanate
}

Wenhao Sun, Weslley G. D. P. Silva and Jennifer van Wijngaarden*

Department of Chemistry, University of Manitoba, Winnipeg, Manitoba, R3T 2N2, Canada

*Corresponding author

Email: vanwijng@cc.umanitoba.ca

Phone: (204)474-8379

Fax: (204)474-7608 


\begin{abstract}
The pure rotational spectra of phenyl isocyanate $(\mathrm{PhNCO})$ and phenyl isothiocyanate (PhNCS) were investigated using Fourier transform microwave (FTMW) spectroscopy in the range from 4 to $26 \mathrm{GHz}$. For each molecule, rotational transitions due to the parent species and nine minor isotopologues including seven ${ }^{13} \mathrm{C}$, one ${ }^{15} \mathrm{~N}$ and one ${ }^{18} \mathrm{O} /{ }^{34} \mathrm{~S}$ have been observed in natural abundance. The $\mathrm{r}_{\mathrm{m}}^{(1)}$ geometries were derived from the resulting sets of rotational constants and are consistent with the equilibrium structures $\left(\mathrm{r}_{\mathrm{e}}\right)$ from ab initio calculations performed at the MP2/aug-cc-pVTZ level. NBO and Townes-Dailey analyses were conducted to better understand the electronic structure and geometry of each compound. In the case of PhNCS, the nitrogen atom displays more sp-like character resulting in shorter $\mathrm{C}-\mathrm{N}$ bonds and a larger $\mathrm{CNC}$ angle relative to those of $\mathrm{PhNCO}$.
\end{abstract}




\section{Introduction}

Organic isocyanates $(\mathrm{RN}=\mathrm{C}=\mathrm{O})$ and isothiocyanates $(\mathrm{RN}=\mathrm{C}=\mathrm{S})$ are common intermediates in the formation of natural products due to the electrophilic carbon atom arising from resonance contributions such as $\mathrm{RN}^{-}-\mathrm{C}^{+}=\mathrm{O}^{-}$and $\mathrm{RN}=\mathrm{C}^{+}-\mathrm{O}^{-}$. Through reactions with alcohols and amines, for example, isocyanates are converted into carbamates $(\mathrm{R}(\mathrm{NH}) \mathrm{C}(=\mathrm{O}) \mathrm{OR}$ ') and urea derivatives $\left(\mathrm{R}(\mathrm{NH}) \mathrm{C}(=\mathrm{O})(\mathrm{NH}) \mathrm{R}^{\prime}\right)$ yielding a diverse range of biologically relevant compounds. Even the simplest versions are the subject of deep scientific interest. Species such as $\mathrm{HNCO},{ }_{1}^{1} \mathrm{HNCS}^{2}$ and $\mathrm{CH}_{3} \mathrm{NCO}^{3}$ are known constituents of molecular clouds where their presence and relative abundance in various sources provide important clues for modelling the chemistry of the interstellar medium. For example, the recent detection of the NCO radical, a building block for peptide bond formation, toward the dense cloud L483 may prove significant in understanding the evolution of amino acids in space. ${ }^{4}$

In the laboratory, a number of small molecules of type RNCO and RNCS have been studied using rotational spectroscopy including those, for example, where $\mathrm{R}=\mathrm{H},{ }^{5,6} \mathrm{CH}_{3},{ }^{7} \mathrm{HCC}^{8,9}$ $\mathrm{NC}^{10,11}$ and $\mathrm{H}_{2} \mathrm{C}_{2}{ }^{12,13}$ In addition to providing fingerprints for astronomical detection, the laboratory spectra provide key information about their molecular geometries and barriers to internal motions which allow exploration of subtle differences in the electronic environments. From the experimental geometries, the NCS containing species have larger valence bond angles 
at nitrogen than their oxygen analogs suggesting that the terminal chalcogen atom affects the electronic structure at nitrogen. The largest difference is seen for the ethynyl substituent as the CNC angle increases by $40^{\circ}$ from $\operatorname{HCCNCO}\left(140^{\circ}\right)^{8}$ to $\operatorname{HCCNCS}\left(180^{\circ}\right){ }^{9}$ Curiously, when substituted with the isoelectronic cyano fragment instead, the angle at nitrogen is smaller by $\sim 10 \%$ for $\operatorname{NCNCO}\left(129^{\circ}\right)^{10}$ but by twice that amount for NCNCS $\left(143^{\circ}\right)$ showing that the electronic properties of the R group are also key and not necessarily scale-able. ${ }^{11}$

The extension of these studies to larger organic groups is of interest to explore the nature of their interactions with the NCS and NCO fragments. The phenyl substituted versions are excellent prototypes as the molecules are planar with only one stable conformer. The ground state microwave spectrum of the normal isotopologues of phenyl isocyanate $(\mathrm{PhNCO})^{14,15}$ and phenyl isothiocyanate (PhNCS) $)^{14,16}$ have been previously reported including investigation of the ${ }^{14} \mathrm{~N}$ nuclear quadrupole hyperfine structure. ${ }^{17}$ These studies have confirmed that the molecules are planar with $\mathrm{C}_{\mathrm{s}}$ symmetry in contrast to infrared spectra which were interpreted based on the assumption of $\mathrm{C}_{2 \mathrm{v}}$ symmetry. ${ }^{18}$ Additional transitions due to excited vibrational states were also assigned and in the case of PhNCO, largely attributed to the out-of-plane torsion of the NCO group about the $\mathrm{C}-\mathrm{N}$ bond. ${ }^{15}$ Previous theoretical investigations using density functional theory have predicted low barriers for this torsion with that of PhNCS $(2.7 \mathrm{~kJ} / \mathrm{mol}$ from DFT B3LYP/6$311++\mathrm{G}(\mathrm{d}, \mathrm{p}))^{19}$ being about half that of PhNCO $\left(5.4 \mathrm{~kJ} / \mathrm{mol}\right.$ from DFT B3LYP/6-311G(d,p)) ${ }^{20}$ While this seems to point to a significant difference in the electronic character of the $\mathrm{Ph}-\mathrm{N}$ bond, the calculated vibrational frequencies for this torsion are surprisingly similar, $58 \mathrm{~cm}^{-1}(\mathrm{PhNCS})^{19}$ and $59 \mathrm{~cm}^{-1}(\mathrm{PhNCO}),{ }^{20}$ but have not been observed to date experimentally.

In order to better understand the effect of the terminal chalcogen atom on the bonding and structure of these compounds, we report herein the first microwave spectroscopic study of 
the nine minor isotopologues of $\mathrm{PhNCO}$ and $\mathrm{PhNCS}$ including heavy atom substitution at each carbon, nitrogen, oxygen and sulfur. Rotational spectra were recorded using both chirped pulse ${ }^{21}$ (cp) and Balle-Flygare ${ }^{22}$ Fourier transform microwave (FTMW) instruments and the spectra of both parent species were re-investigated to include $b$-type rotational transitions which were not previously observed due to their low intensities. Their observation in this work has allowed more precise determination of the A rotational constants and thus, enabled accurate experimental structures to be derived for the first time. Analysis of the ten sets of rotational constants of each compound were used to derive the mass dependence structures $\left(\mathrm{r}_{\mathrm{m}}{ }^{(1)}\right)$ which are found to be consistent with the equilibrium structures $\left(r_{e}\right)$ derived at the MP2/aug-cc-pVTZ level of theory. The barrier to both out-of-plane torsion and in-plane bending motions involving the NCX fragment were calculated and subtle differences in the electronic structure were explored using natural bond orbital (NBO) calculations and Townes-Dailey analysis.

\section{Experimental}

Commercial samples of PhNCO and PhNCS (both 98.0\% purchased from Sigma-Aldrich Canada) were used without further purification. As both samples are liquids at room temperature (mp: $-30^{\circ} \mathrm{C}$ for $\mathrm{PhNCO}$ and $-21^{\circ} \mathrm{C}$ for PhNCS) with relatively high boiling points (bp: 162-163 ${ }^{\circ} \mathrm{C}$ for $\mathrm{PhNCO}$ and $218{ }^{\circ} \mathrm{C}$ for PhNCS), they were placed into glass bubblers separately through which a carrier gas (neon at $\sim 1$ bar) was used to deliver the samples into the spectrometers. The sample mixtures were expanded into the high vacuum chambers of the spectrometers via a supersonic jet expansion using a pulsed nozzle. The rotational spectra of the compounds were recorded using both chirped pulse and cavity-based FTMW instruments, which have been described in detail elsewhere. ${ }^{23,24}$ Analysis of the broadband cp-FTMW spectra, acquired in 
segments of $2 \mathrm{GHz}$ in the frequency range from 8 to $18 \mathrm{GHz}$, allowed the assignment of the most intense rotational transitions for both $\mathrm{PhNCO}$ and PhNCS corresponding to the parent species and their minor isotopologues $\left({ }^{13} \mathrm{C},{ }^{15} \mathrm{~N},{ }^{18} \mathrm{O}\right.$ and $\left.{ }^{34} \mathrm{~S}\right)$. A $140 \mathrm{MHz}$ portion of the broadband spectrum of PhNCO is provided in Figure 1 as an example. Next, aiming to achieve higher resolution and sensitivity, and in order to analyze the hyperfine structure due to the presence of the ${ }^{14} \mathrm{~N}$ nuclei in the compounds, individual rotational transitions were measured in the range of 4-26 GHz using the cavity-based FTMW spectrometer. A sample spectrum of the $66_{16}-5_{15}$ rotational transition of PhNCO is shown in Figure 2. The observed transitions in the cavity-based spectra are split into two due to the Doppler effect and typically have line widths of $\sim 7 \mathrm{kHz}$. The uncertainty in the line positions is about $\pm 1 \mathrm{kHz}$.

Figure 1. $140 \mathrm{MHz}$ portion of the cp-FTMW spectrum collected with 1.5 million FIDs showing the relative intensity of the rotational transition $6_{06}-5_{05}$ for the parent $\mathrm{PhNCO}$ species, six ${ }^{13} \mathrm{C}$ and one ${ }^{15} \mathrm{~N}$ minor isotopologues. 


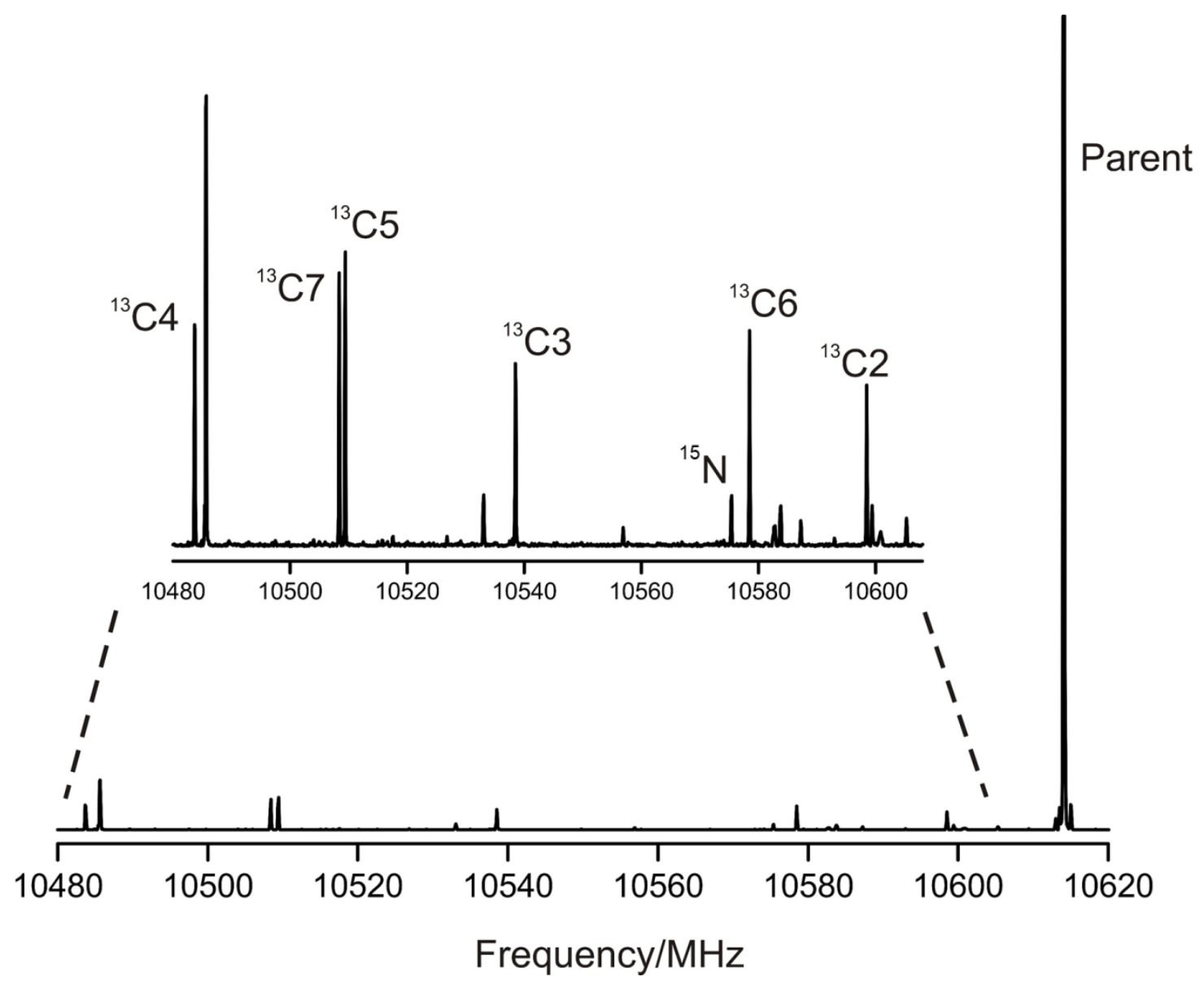

Figure 2. Sample of FTMW spectrum (800 cycles) of the $66_{16}-5_{15}$ rotational transitions of PhNCO showing the ${ }^{14} \mathrm{~N}$ hyperfine structure. 


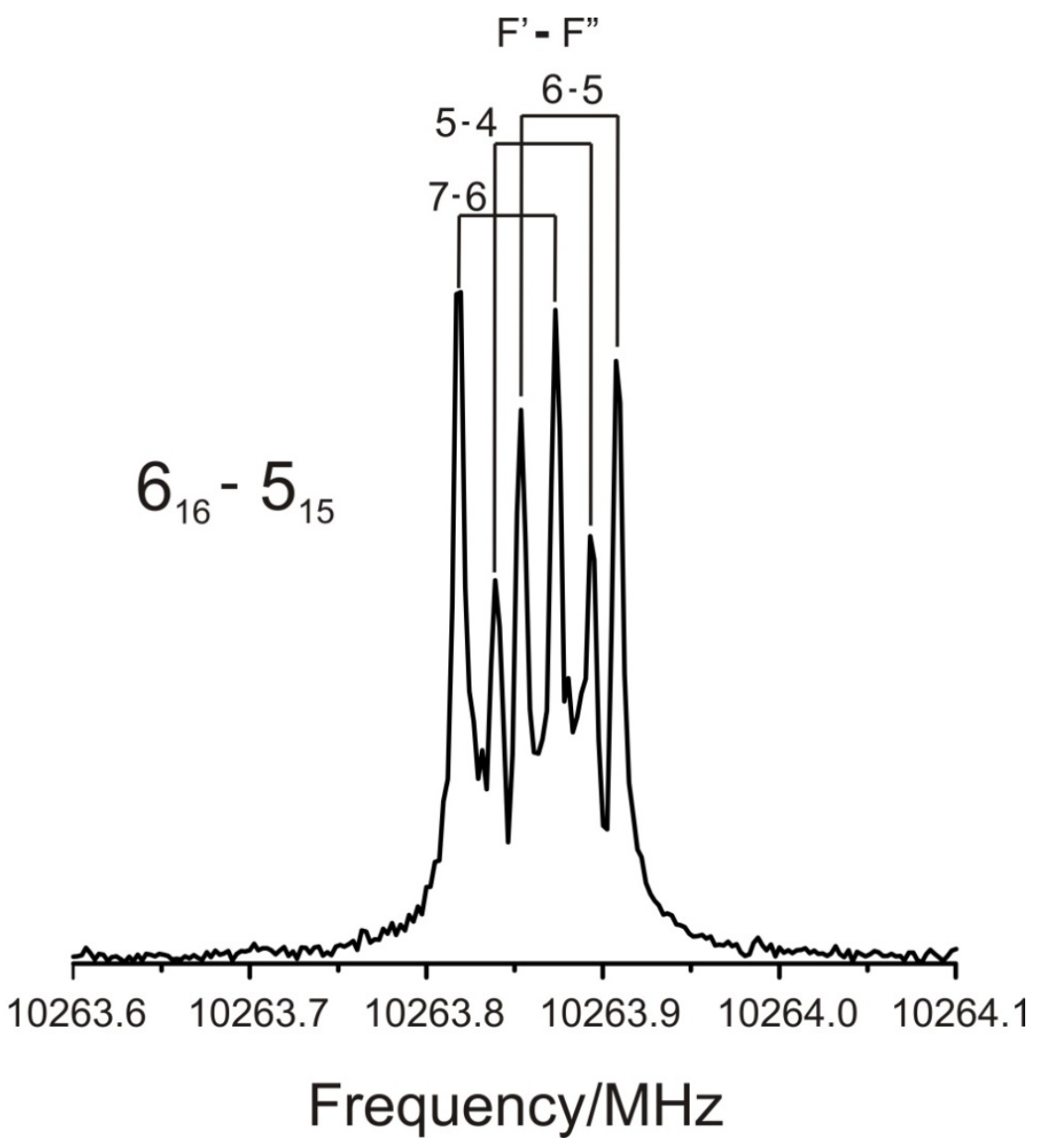

\section{Computational}

The geometries of the compounds were optimized using the MP2, B3LYP and B3LYPD3BJ methods combined with the aug-cc-pVTZ basis set in the Gaussian 16 Revision B.01 program. ${ }^{25}$ Frequency calculations were done at these levels of theory to verify that the resulting geometries corresponded to energy minima and to estimate the low frequency modes. From the optimized structures, the rotational constants, electric dipole moments and ${ }^{14} \mathrm{~N}$ quadrupole coupling constants were extracted to assist with the interpretation of the experimental rotational spectra. The resulting equilibrium structures are shown in their principal axis systems in Figure 3 
and the Cartesian coordinates are given in the supplementary information for all three levels of theory employed.

Although PhNCO and PhNCS are known to be planar with $\mathrm{C}_{\mathrm{s}}$ symmetry, the previous infrared work based on $\mathrm{C}_{2 \mathrm{v}}$ symmetry along with the recent observation of linear HCCNCS, ${ }^{9}$ $\mathrm{HCCCCNCS}^{26}$ and $\mathrm{NCCCNCS}^{26}$ inspired us to look more closely at the energy profile corresponding to the lowest energy in-plane and out-of-plane modes that correspond to bending of the $\angle \mathrm{C} 1 \mathrm{NC} 7$ angle and torsion about the $\mathrm{C} 1-\mathrm{N}$ bond. These motions interconvert the compounds between two equivalent planar forms. To better understand the energy barrier associated with these re-arrangements, potential energy scans were performed at each level of theory. In the first scan, $\angle \mathrm{C} 1 \mathrm{NC} 7$ was varied from $120^{\circ}$ to $180^{\circ}$ in steps of $3^{\circ}$ whereas in the second one, the dihedral angle $\mathrm{C} 2-\mathrm{C} 1-\mathrm{N}-\mathrm{C} 7$ was rotated from $0^{\circ}$ to $90^{\circ}$ in steps of $10^{\circ}$. During the scan calculations, all other geometrical parameters were relaxed. When the dihedral angle was set to $90^{\circ}$ in PhNCS, the valence angle $\angle \mathrm{C} 1 \mathrm{NC} 7$ increased to move the NCS group into the plane of the ring resulting in the same energy as reported at $180^{\circ}$ for $\angle \mathrm{C} 1 \mathrm{NC} 7$ in the first scan. The resulting potential energy plots are shown in Figure 4.

Finally, we also performed natural bond orbital (NBO) analysis on the geometry that was optimized at the MP2/aug-cc-pVTZ level to gain greater insight into differences in the electronic structure of PhNCO and PhNCS and how these influence their geometries. The NBO calculations were carried out using the NBO 6.0 routine $^{27}$ at the B3LYP/aug-cc-pVTZ level. The output is included in the supplementary information. 
Figure 3. Equilibrium geometries of PhNCO and PhNCS in the principal axis system obtained at the MP2/aug-cc-pVTZ level of theory.
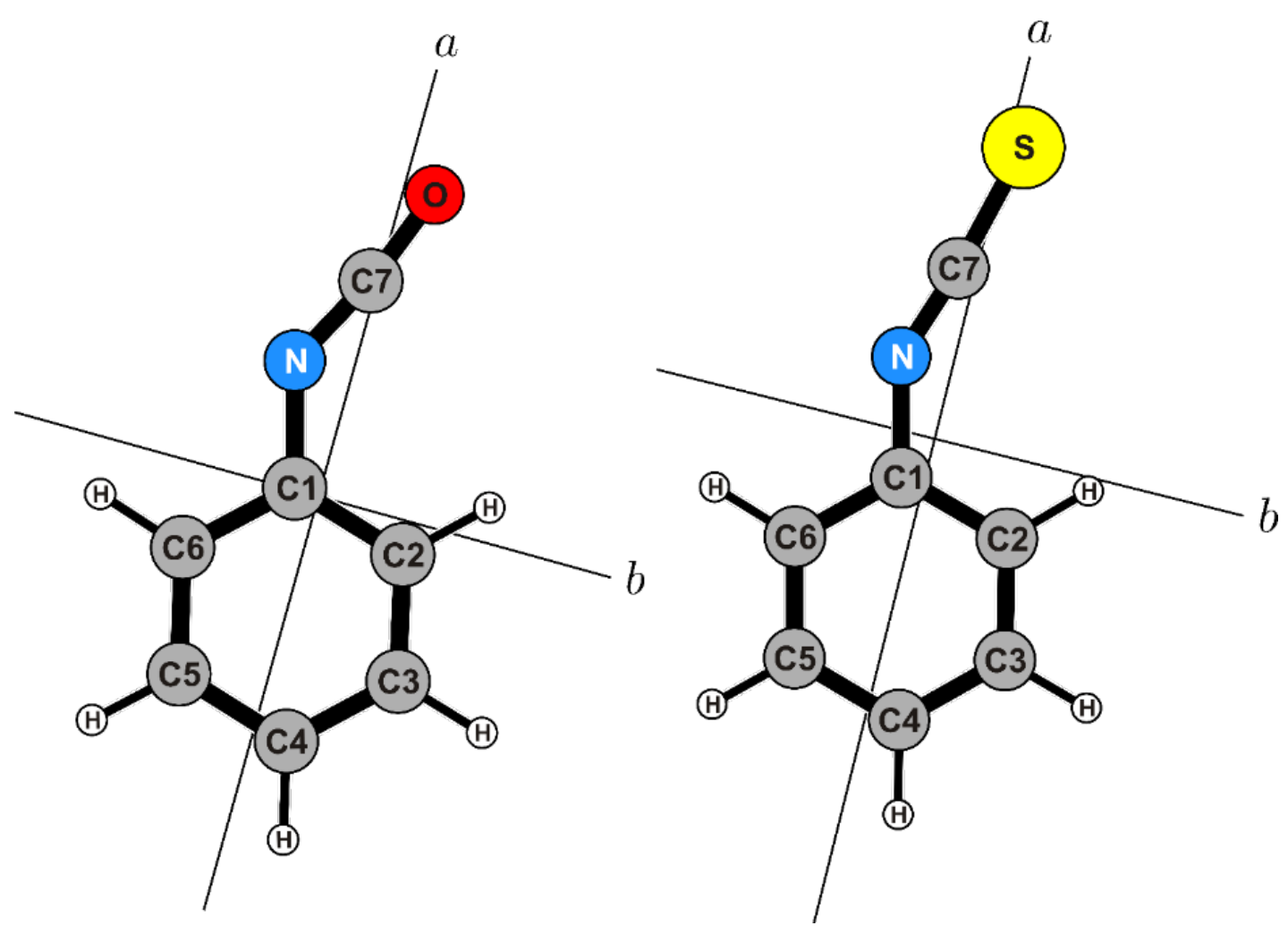
Figure 4. Potential energy curves for $\mathrm{C} 2-\mathrm{C} 1-\mathrm{N}-\mathrm{C} 7$ dihedral angle and $\angle \mathrm{C} 1 \mathrm{NC} 7$ for $\mathrm{PhNCO}$ and PhNCS.

$\mathrm{PhNCO}$
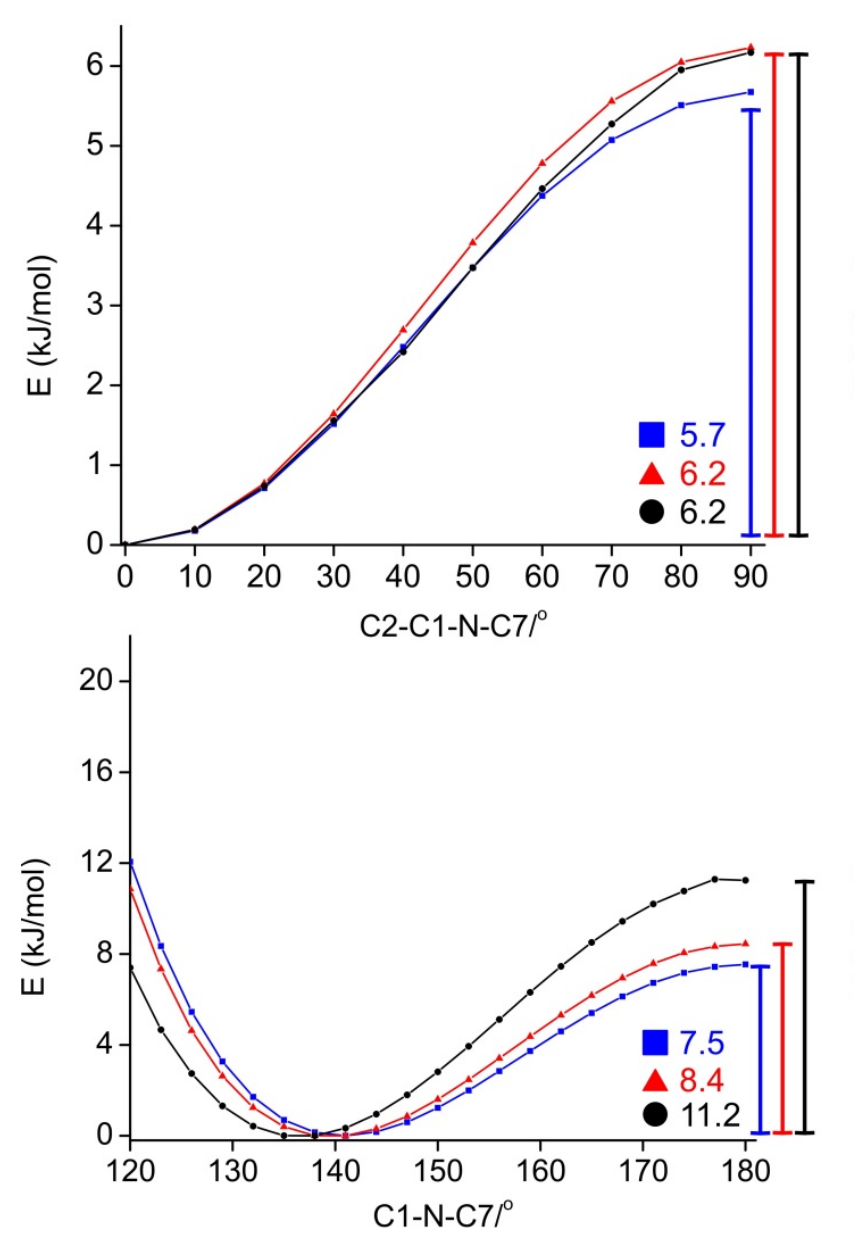

PhNCS
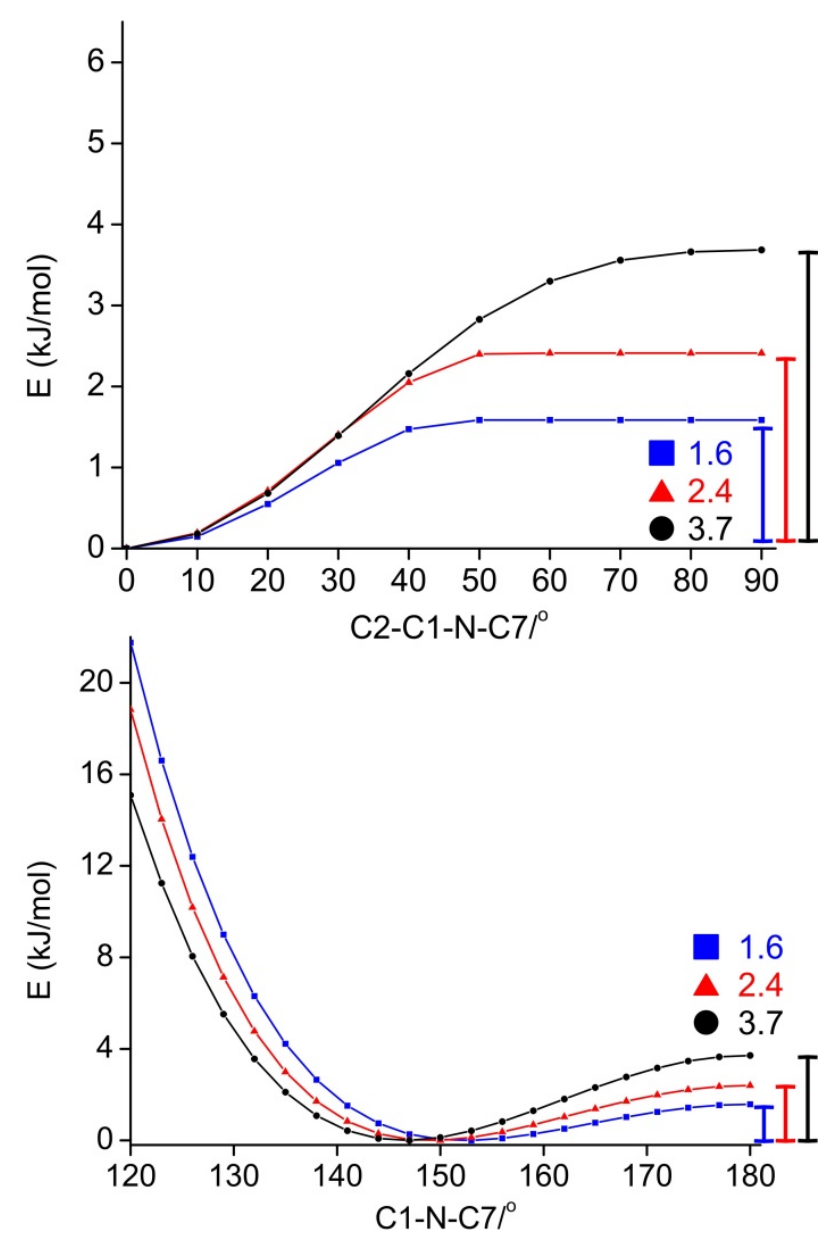

B3LYP $\triangle$ B3LYP-D3BJ • MP2 


\section{$\underline{\text { Spectral analysis }}$}

The theoretical calculations predict $\mathrm{PhNCO}$ to have sizeable electric dipole moment components of $\left|\mu_{\mathrm{a}}\right|=2.77 \mathrm{D}$ and $\left|\mu_{\mathrm{b}}\right|=0.28 \mathrm{D}$ (MP2/aug-cc-pVTZ) which are consistent with previous experimental results from Stark measurements $\left|\mu_{\mathrm{a}}\right|=2.50 \pm 0.02 \mathrm{D}$ and $\left|\mu_{\mathrm{b}}\right|<0.2 \mathrm{D}{ }^{15}$ Based on this, the rotational spectrum of this species is dominated by $a$-type rotational transitions. Indeed, a total of 244 rotational transitions including $208 a$-type and $36 b$-type, ranging from $\mathrm{J}^{\prime}=3-11$ were observed for the parent of $\mathrm{PhNCO}$. The intensities were sufficient to proceed with the identification of $30 a$-type rotational transitions corresponding to the ${ }^{15} \mathrm{~N}$ isotopologue, 41 transitions due to the ${ }^{18} \mathrm{O}$ isotopologue, and more than 60 transitions for each of the six ${ }^{13} \mathrm{C}$ isotopologues. For the ${ }^{13} \mathrm{C} 1$ isotopologue, as the position of $\mathrm{C} 1$ is close to the center of mass, most of the rotational transitions are overshadowed by the analogous transition of the parent species and thus, only 18 lines were measured. A full list of the observed transitions is provided as supplementary material.

PhNCS is predicted to have dipole moment components of $\left|\mu_{\mathrm{a}}\right|=3.15 \mathrm{D}$ and $\left|\mu_{\mathrm{b}}\right|=0.29 \mathrm{D}$ (MP2/aug-cc-pVTZ) which indicates that the rotational spectrum of this species is also dominated by $a$-type rotational transitions. In total, 214 rotational transitions, including 189 $a$-type and $25 b$-type, ranging from $\mathrm{J}^{\prime}=2-15$ were measured for the parent species. The intensities of the observed transitions were sufficient to proceed with the identification of $65 a$ type rotational transitions corresponding to the ${ }^{34} \mathrm{~S}$ isotopologue, 20 due to the ${ }^{15} \mathrm{~N}$ isotopologue, and more than 27 for each of the seven ${ }^{13} \mathrm{C}$ isotopologues. A list of the observed lines for PhNCS is also provided as supplementary material.

The rotational, centrifugal distortion, and ${ }^{14} \mathrm{~N}$ nuclear quadrupole coupling constants were determined for each species by fitting the observed rotational transitions using Pickett's SPFIT program $^{28}$ set to Watson's A-reduced Hamiltonian. ${ }^{29}$ The results for PhNCO and PhNCS are 
shown in Tables 1 and 2, respectively. As fewer lines were observed for the minor isotopologues, their centrifugal distortion constants were fixed at the parent value in cases where a specific parameter was not well-determined after running a preliminary fit of the data. For those constants that were not fixed, for example $\Delta_{\mathrm{J}}$ for all species, the values obtained from the fits are similar to those of the parent. This confirms the validity of the common assumption that centrifugal distortion is not significantly different upon isotopic substitution in these systems.

\section{Structural determination}

Based on ab initio calculations, both $\mathrm{PhNCO}$ and PhNCS have planar equilibrium structures with $\mathrm{C}_{\mathrm{s}}$ molecular symmetry. In principle, with 10 isotopologues observed for each, the experimental rotational constants can be used to derive substitution structures $\left(\mathrm{r}_{\mathrm{s}}\right)$ through a Kraitchmann analysis. ${ }^{30}$ In practice, as the a-axis passes close to $\mathrm{C} 1$ and $\mathrm{C} 7$ in both molecules, the positions of these atoms are not well-determined by this method and instead, mass dependence structures $\left(\mathrm{r}_{\mathrm{m}}{ }^{(1)}\right)$ as described by Watson ${ }^{31}$ were derived as shown for other benzene derivatives. ${ }^{32,33}$ The 30 rotational constants from the set of 10 isotopologues were used in Kisiel's STRFIT ${ }^{34}$ least squares fitting routine to estimate key geometric parameters for the heavy atoms while internal parameters involving the $\mathrm{H}$ atoms were held fixed at the values obtained from MP2/aug-cc-pVTZ calculations. To preserve the orientation of the $\mathrm{H}$ atoms relative to the ring, the difference of the external angles (for example $\angle \mathrm{HC} 2 \mathrm{C} 1-\angle \mathrm{HC} 2 \mathrm{C} 3$ ) of each $\mathrm{H}$ were also held at the ab initio values following the example of substituted benzonitriles. ${ }^{35}$

The Laurie parameter $\delta_{\mathrm{H}}$ was fixed at $0.01 \AA$ for each $\mathrm{CH}$ bond and $\mathrm{c}_{\mathrm{a}}=\mathrm{c}_{\mathrm{b}}=\mathrm{c}_{\mathrm{c}}$ was assumed as done previously for benzaldehyde. ${ }^{32}$ A summary of the results is provided in Table 3 under the $\mathrm{r}_{\mathrm{m}}{ }^{(1)}$ heading along with the ab initio values for the equilibrium structure $\left(\mathrm{r}_{\mathrm{e}}\right)$ for comparison. 


\section{$\underline{\text { Discussion }}$}

The spectroscopic parameters for $\mathrm{PhNCO}$ and PhNCS are well-determined and are compared with the calculated values at the MP2/aug-cc-pVTZ and B3LYP-D3BJ/aug-cc-pVTZ levels of theory in Table 4. For the rotational and ${ }^{14} \mathrm{~N}$ quadrupole coupling constants, both methods provide reasonable agreement to the experimental values but the MP2 derived parameters yield a closer match for the rotational constants and $\chi_{\text {aa. }}$ The experimental values of $\chi_{\mathrm{bb}}$ and $\chi_{\mathrm{cc}}$ seemingly align better with the B3LYP-D3BJ estimates but one should make this comparison with caution as the actual fit parameter $\left(0.25\left(\chi_{b b}-\chi_{c c}\right)\right)$ was a small number. To test the influence of this small parameter on the spectral pattern, we simulated the nuclear quadrupole hyperfine structure based on each set of coupling constants to verify that the resulting patterns were comparable. An additional check comes from comparing the experimental values determined in this work with those of the previous study of Kasten and Dreizler ${ }^{17}$ as shown below Tables 1 and 2. Owing to the observation of many new transitions (including $b$-type transitions for the first time), higher resolution, and consistency across all 10 isotopologues for each compound, we are confident in our assignments.

The centrifugal distortion constants of PhNCO and PhNCS have also been determined at both the MP2/aug-cc-pVTZ and B3LYP-D3BJ/aug-cc-pVTZ levels of theory and are summarized in Table 4. Although harmonic frequency calculations provide very reliable estimates of these parameters for similarly sized ring molecules such as anisole and benzaldehyde using both MP2 and DFT methods $(6-31 \mathrm{G}(\mathrm{d}, \mathrm{p})),{ }^{32}$ the results for PhNCO and PhNCS in this work show larger discrepancies between experiment and theory with $\Delta_{\mathrm{JK}}$ differing by more than an order of magnitude from the experimental values. Parameters derived from anharmonic frequency calculations, also included in Table 4, performed significantly better as 
one might expect for a molecule with low frequency vibrations. For comparison, a recent study by Alonso et al. ${ }^{36}$ on $\alpha$-aminoisobutyric acid revealed even larger deviations between experimental centrifugal distortion constants and those calculated at the MP2/6-311++G(d,p) level (harmonic) for one conformer. They attributed this to the low barrier $\left(85 \mathrm{~cm}^{-1}\right)$ for the $\mathrm{C}_{\alpha^{-}}$ $\mathrm{COOH}$ torsional motion which interconverts between two equivalent forms and they successfully modelled the effect using a quartic potential to explain their anomalously large centrifugal distortion constants. In the current study, the analogous torsional barriers are much higher as shown in Figure $4\left(520 \mathrm{~cm}^{-1}\right.$ for PhNCO and $309 \mathrm{~cm}^{-1}$ for PhNCS) and as such, the quantum chemical values in Table 4 provide reasonable estimates.

The inertial defects $\left(\Delta_{0}\right)$ derived from the experimental rotational constants for the parent species (-0.132 amu $\AA^{2}$ for PhNCO and $0.101 \mathrm{amu} \cdot \AA^{2}$ for PhNCS) are small as expected for planar molecules but non-zero due to contributions from low frequency vibrations. For PhNCO, the negative value suggests that these motions are largely out-of-plane which is consistent with frequency calculations showing that the torsion about C1-N to twist the NCO group out-of-plane falls at $57 \mathrm{~cm}^{-1}$ (MP2/aug-cc-pVTZ). The next most energetic mode corresponds to $\angle \mathrm{C} 1 \mathrm{NC} 7$ bending $\left(91 \mathrm{~cm}^{-1}\right)$ and is considerably higher in energy. For PhNCS, in contrast, these two modes are similar in energy $\left(54 \mathrm{~cm}^{-1}\right.$ and $58 \mathrm{~cm}^{-1}$, respectively) and the in-plane motion must play a larger role due to the positive value of the inertial defect. The increased energy for the in-plane bending motion of $\mathrm{PhNCO}$ compared with the out-of-plane torsion appears consistent with the trend observed in the potential energy curves in Figure 4. For the in-plane re-arrangement, the barrier to linearity at nitrogen is $11.2 \mathrm{~kJ} / \mathrm{mol}$ while the barrier for the out-of-plane torsion of the $\mathrm{NCO}$ group is considerably lower at $6.2 \mathrm{~kJ} / \mathrm{mol}$. For PhNCS, the barrier to interconvert between the equivalent planar structures is lower $(3.7 \mathrm{~kJ} / \mathrm{mol})$ and actually passes through the same $\mathrm{C}_{2 \mathrm{v}}$ 
geometry for both pathways (even when the C2-C1-N-C7 dihedral angle is set to $90^{\circ}$ ) when the other geometric parameters are relaxed in the 1D scans. These differences suggest that the terminal chalcogen atom has an important influence on the electronic structure around C1-N.

Using the rotational constants from all 10 isotopologues of PhNCO and PhNCS, the experimental geometries $\left(\mathrm{r}_{\mathrm{m}}^{(1)}\right)$ of the heavy atoms have been precisely determined. This assertion is based upon comparison of the 30 experimentally determined rotational constants from each isotopologue of PhNCO and PhNCS with those derived based on the $\mathrm{r}_{\mathrm{m}}{ }^{(1)}$ geometry determined in the fit. The maximum discrepancy between the observed and calculated rotational constants is less than $0.015 \%$ for $\mathrm{A}$ and an order of magnitude lower for B and C in each set. The geometric parameters are summarized along with the equilibrium $\left(\mathrm{r}_{\mathrm{e}}\right)$ structural parameters from MP2/aug-cc-pVTZ calculations in Table 3 and from comparison, it is clear that the same trends are captured in both sets of results. The parameters that govern the phenyl ring geometry show no discernable change when oxygen is replaced by sulfur which suggests that the identity of the terminal atom does not change the nature of bonding in the ring itself through delocalization via the $\pi$-electrons for example. This is supported by the NBO results which show that the natural charges for $\mathrm{C} 1$ through $\mathrm{C} 6$ are similar in both compounds and that there are no clearly different perturbative interactions that preferentially add or remove electron density from the ring. This is also consistent with the observation of low torsional barriers reported for these and related compounds such as the ketene and azide substituted benzenes. ${ }^{20}$

The terminal atom, however, has a greater effect on the geometry at nitrogen. With recognition that the experimental uncertainties make it difficult to declare that small changes in parameters are meaningful, the trends for both the $r_{m}^{(1)}$ and $r_{e}$ structures reveal that the largest changes in bond lengths are those involving nitrogen $(\mathrm{NC} 1, \mathrm{C} 7 \mathrm{~N})$. These shorten by $\sim 0.01 \AA$ in 
PhNCS compared with PhNCO. This shortening is accompanied by an increase in the $\angle \mathrm{C} 1 \mathrm{NC} 7$ by $\sim 10^{\circ}$ which suggests that it is the electronic structure at nitrogen atom that is most affected when replacing oxygen with sulfur. The increase in this angle at nitrogen is similar to that observed in the $\mathrm{HNCO}\left(128^{\circ}\right)^{5} / \mathrm{HNCS}\left(136^{\circ}\right)^{6}$ and $\mathrm{CH}_{3} \mathrm{NCO}\left(140^{\circ}\right) / \mathrm{CH}_{3} \mathrm{NCS}\left(147.5^{\circ}\right)^{7}$ pairs.

To better understand these geometric changes, the nature of the occupied orbitals for PhNCO and PhNCS was considered and the most striking difference is that there is an orbital identified as a lone pair for nitrogen in the former but not in the latter. Comparison of the hybridization of the nitrogen atoms reveals that for PhNCS, the electronic structure is that of a sp hybrid orbital while for PhNCO, it is more like $\mathrm{sp}^{1.6}$. This implies the addition of triple bond character in the former which is confirmed by the appearance of a second $\pi$ molecular orbital between $\mathrm{N}$ and $\mathrm{C} 7$ in this compound. Closer inspection of the NBO results shows, however, that this orbital is $85 \%$ localized on nitrogen and thus approximates lone pair character at that site with some degree of delocalization through the additional $\pi$ bond with $\mathrm{C} 7$. This subtle difference in the nature of the orbitals involving nitrogen is in line with the observed geometry differences in $\mathrm{PhNCO}$ and PhNCS and also explains why the latter retains $\mathrm{C}_{\mathrm{s}}$ symmetry rather than adopt $\mathrm{C}_{2 \mathrm{v}}$ symmetry.

The experimentally derived ${ }^{14} \mathrm{~N}$ quadrupole coupling constants of PhNCO and PhNCS can be used to derive an independent estimate of the electronic structure at that site using a Townes-Dailey analysis as described in detail by Gordy and $\mathrm{Cook}^{37}$ and as applied to other nitrogen containing species such as the fluoropyridines. ${ }^{38,39}$ To obtain the quadrupole coupling constants in the quadrupole tensor frame $(\mathrm{x}, \mathrm{y}, \mathrm{z})$, the principal inertial system was rotated by $7.3^{\circ}$ and $3.3^{\circ}(a \rightarrow x, b \rightarrow z, c \rightarrow y)$ through the QDIAG program ${ }^{34}$ for PhNCO and PhNCS, respectively. As the off-diagonal term ( $\left.\chi_{\mathrm{ab}}\right)$ was not well-determined, it was fixed at the ab initio 
value (-0.545 MHz for PhNCO and 0.195 MHz for PhNCS) at the MP2/aug-cc-pVTZ level.

From reference 37 , the $2 \mathrm{~s}, 2 \mathrm{p}_{\mathrm{x}}, 2 \mathrm{p}_{\mathrm{y}}, 2 \mathrm{p}_{\mathrm{z}}$ orbital populations of nitrogen are:

$$
\begin{gathered}
\mathrm{n}_{2 \mathrm{~s}}=\mathrm{n}_{\psi_{1}}\left(1-2 \alpha_{\mathrm{s}}^{2}\right)+\left(1+\mathrm{i}_{\sigma}\right) \times 2 \alpha_{\mathrm{s}}^{2} \\
\mathrm{n}_{2 \mathrm{p}_{\mathrm{z}}}=\mathrm{n}_{\Psi_{1}} 2 \alpha_{\mathrm{s}}^{2}+\left(1+\mathrm{i}_{\sigma}\right) \times\left(1-2 \alpha_{\mathrm{s}}^{2}\right) \\
\mathrm{n}_{2 \mathrm{p}_{\mathrm{x}}}=1+\mathrm{i}_{\sigma} \\
\mathrm{n}_{2 \mathrm{p}_{\mathrm{y}}}=1+\mathrm{i}_{\pi}
\end{gathered}
$$

where $n_{\psi 1}$ is the occupancy of the lone pair orbital, $i_{\sigma}$ is the ionic character of the C-N $\sigma$-bonds, $i_{\pi}$ is that of the C-N $\pi$-bond, and $\alpha_{\mathrm{s}}{ }^{2}$ is the s character in the hybrid orbitals. As only two independent parameters are known experimentally $\left(\chi_{z z}, \chi_{x x}-\chi_{y y}\right)$, one can only derive two of these quantities related to the electronic structure. Assuming that the ionic character $\mathrm{i}_{\sigma}$ across the C-N bonds is described by the difference in electronegativities of $\mathrm{C}$ and $\mathrm{N}: \mathrm{i}_{\sigma(\mathrm{NC} 1)}=\mathrm{i}_{\sigma(\mathrm{NC} 1)}=\mid \mathrm{x}_{\mathrm{C}}-$ $\mathrm{x}_{\mathrm{N}} / 2=0.25$ as invoked in reference 37 and estimating $\alpha_{\mathrm{s}}{ }^{2}$ from the experimentally derived CNC angle as in reference 38 the nuclear quadrupole coupling constants can be used to derive $n_{\psi 1}$ and $\mathrm{i}_{\pi}$ from the following relationships:

$$
\begin{aligned}
& \chi_{z z}=\left(n_{2 p_{z}}-\frac{n_{2 p_{x}}+n_{2 p_{y}}}{2}\right) \times\left(\frac{e Q q_{210}(N)}{1+\varepsilon c^{-}}\right)=-1.517 \mathrm{MHz} \\
& \chi_{x x}-\chi_{y y}=\frac{3}{2}\left(n_{2 p_{x}}-n_{2 p_{y}}\right) \times\left(\frac{e Q q_{210}(N)}{1+\varepsilon c^{-}}\right)=4.071 \mathrm{MHz}
\end{aligned}
$$

where $\mathrm{eQq}_{210}(\mathrm{~N})=-11.2 \mathrm{MHz}$ is the atomic orbital coupling of a p-electron on nitrogen, ${ }^{40}$ $\varepsilon=0.30$ is the charge screening correction for a nitrogen p-orbital, and the negative charge on nitrogen can be calculated in units of $e$ from $\mathrm{c}^{-}=2 \mathrm{i}_{\sigma}+\mathrm{i}_{\pi}-\left(2-\mathrm{n}_{\psi 1}\right) \cdot{ }^{37} \mathrm{~A}$ summary of the results from the analysis is provided in Table 5 along with results from the NBO calculations for 
comparison. Based on the agreement of the various parameters, it is evident that the TownesDailey approach, as a simplified model based on valence bond theories, is a valid tool to estimate the electronic structure around nitrogen. In comparison to $\mathrm{PhNCO}$, the smaller charge on nitrogen and the lower population in the nitrogen lone pair orbital in PhNCS from the TownesDailey results are indicative of greater delocalization of the electron density around the nitrogen center in the latter. This is, of course, consistent with the above discussion of the NBO results but direct comparison of values between these two methods should be done with caution due to differences in the models from which they are derived.

The role of the terminal chalcogen atoms in the subtle changes inferred about the electron density at nitrogen is not straightforward to extract due to their differences in atomic size, electronegativity and hybridization. The natural charge on oxygen $(-0.485)$ indicates greater electron density at the end of the NCO fragment than in the sulfur (0.029) analog as expected. This is reflected in differences in the nature of bonding within the NCO/NCS moieties in general. The C7S $\sigma$-bond in PhNCS, for example, is characterized by a more even sharing of electrons $(48 \% \mathrm{C} 7: 52 \% \mathrm{~S})$ and the hybrid orbital on sulfur has significantly enhanced $\mathrm{p}$-character $\left(\mathrm{sp}^{6}\right)$ in comparison to $\mathrm{PhNCO}\left(35 \% \mathrm{C} 7: 65 \% \mathrm{O}, \mathrm{sp}^{2.2}\right)$. There are notable differences in the nature of the $\pi$-bonding and lone pair characters of the chalcogen atoms also and collectively, these alter the electronic structure of the NCX fragment in PhNCO and PhNCS sufficiently to lead to the observed geometric changes at nitrogen.

In summary, the rotational spectra of both PhNCO and PhNCS are reported for the parent and nine minor isotopologues. By using the rotational constants for all heavy atom isotopologues, the experimental $\left(\mathrm{r}_{\mathrm{m}}{ }^{(1)}\right)$ geometries were obtained and observed to be in good agreement with $a b$ initio $r_{e}$ geometries at the MP2/aug-cc-pVTZ level. The greatest difference in the geometries 
occurs due to changes in the hybridization of the nitrogen atom favouring a more linear, sp-like structure in the S-containing species. From the derived geometries, this change results in shorter C-N bonds and a larger valence bond angle at nitrogen in PhNCS compared with PhNCO. Finally, the derived $\mathrm{C} 1-\mathrm{N}$ and $\mathrm{C} 7-\mathrm{N}$ bond lengths and the $\angle \mathrm{C} 1 \mathrm{NC} 7$ angles mirror those predicted ${ }^{41}$ for vinyl-NCO and vinyl-NCS suggesting that the hybridization of the adjoining atom of the R group has a critical influence on the electronic structure at nitrogen.

\section{Supporting information}

Appendix I: Equilibrium Structures from MP2/aug-cc-pVTZ, B3LYP/aug-cc-pVTZ, B3LYPD3BJ/aug-cc-pVTZ Calculations for PhNCO and PhNCS

Appendix II: Assigned Transitions for PhNCO and PhNCS and Their Minor Isotopologues Appendix III: Kraitchman Coordinates for PhNCO and PhNCS

Appendix IV: NBO Output for PhNCO and PhNCS

\section{$\underline{\text { Acknowledgements }}$}

This research is funded by the Natural Sciences and Engineering Research Council of Canada (NSERC) through the Discovery Grant program and the University of Manitoba for providing access to its advanced research computing resource, Grex. W. Sun and W. Silva are grateful for financial support provided through the GETS and UM Graduate Fellowship (UMGF) programs through the Faculty of Graduate Studies of the University of Manitoba. 
Table 1. Ground State Spectroscopic Constants and Standard Errors Obtained for PhNCO and Its Isotopologues.

\begin{tabular}{|c|c|c|c|c|c|c|c|c|c|c|}
\hline & $\mathrm{PhNCO}$ & ${ }^{13} \mathrm{C} 1$ & ${ }^{13} \mathrm{C} 2$ & ${ }^{13} \mathrm{C} 3$ & ${ }^{13} \mathrm{C} 4$ & ${ }^{13} \mathrm{C} 5$ & ${ }^{13} \mathrm{C} 6$ & ${ }^{13} \mathrm{C} 7$ & ${ }^{15} \mathrm{~N}$ & ${ }^{18} \mathrm{O}$ \\
\hline \multicolumn{11}{|c|}{ Rotational Constants $^{a} / \mathrm{MHz}$} \\
\hline$A$ & $5201.7138(3)$ & $5197.76(2)$ & $5142.347(7)$ & $5101.517(9)$ & $5194.081(9)$ & $5154.498(9)$ & $5114.580(8)$ & $5201.243(9)$ & $5178.363(5)$ & $5188.98(2)$ \\
\hline$B$ & $972.68164(2)$ & $972.714(1)$ & $972.46023(7)$ & $967.23964(8)$ & $959.45580(8)$ & $962.9936(1)$ & $971.06000(9)$ & $961.82997(8)$ & $969.23042(6)$ & $929.1584(1)$ \\
\hline$C$ & $819.62560(2)$ & $819.5501(4)$ & $817.98219(4)$ & $813.24982(6)$ & $810.03060(6)$ & $811.57503(6)$ & $816.28202(6)$ & $811.89565(6)$ & $816.59922(5)$ & $788.2085(1)$ \\
\hline \multicolumn{11}{|c|}{ Centrifugal Distortion Constants ${ }^{b} / \mathrm{kHz}$} \\
\hline$\Delta_{J}$ & $0.07264(9)$ & $0.074(2)$ & $0.0726(4)$ & $0.0732(4)$ & $0.0713(4)$ & $0.0692(5)$ & $0.0715(4)$ & $0.0709(4)$ & $0.0710(2)$ & $0.0699(9)$ \\
\hline$\Delta_{J K}$ & $-0.2403(5)$ & {$[-0.2403]$} & $-0.20(1)$ & $-0.26(1)$ & $-0.28(1)$ & $-0.19(1)$ & $-0.22(1)$ & $-0.24(1)$ & $-0.292(3)$ & $-0.27(2)$ \\
\hline$\Delta_{K}$ & $3.39(6)$ & {$[3.39]$} & {$[3.39]$} & {$[3.39]$} & {$[3.39]$} & {$[3.39]$} & {$[3.39]$} & [3.39] & {$[3.39]$} & {$[3.39]$} \\
\hline$\delta_{J}$ & $0.01669(7)$ & $0.018(2)$ & $0.0162(3)$ & $0.0170(3)$ & $0.0166(3)$ & $0.0153(4)$ & $0.0165(3)$ & $0.0158(3)$ & $0.0161(2)$ & $0.0168(9)$ \\
\hline$\delta_{K}$ & $0.451(7)$ & {$[0.451]$} & {$[0.451]$} & {$[0.451]$} & {$[0.451]$} & {$[0.451]$} & {$[0.451]$} & {$[0.451]$} & {$[0.451]$} & {$[0.451]$} \\
\hline \multicolumn{11}{|c|}{${ }^{14} \mathrm{~N}$ Quadrupole Coupling Constants /MHz } \\
\hline$\chi_{\mathrm{aa}}$ & $2.7241(4)$ & $2.8(1)$ & $2.72(1)$ & $2.72(1)$ & $2.73(1)$ & $2.74(1)$ & $2.74(1)$ & $2.73(1)$ & - & $2.71(1)$ \\
\hline$\chi_{\mathrm{bb}}$ & $-1.4474(4)$ & {$[-1.4714]$} & $-1.45(1)$ & $-1.43(1)$ & $-1.45(1)$ & $-1.44(1)$ & $-1.45(1)$ & $-1.44(1)$ & - & $-1.42(1)$ \\
\hline$\chi_{c c}$ & $-1.2766(4)$ & {$[-1.3006]$} & $-1.28(1)$ & $-1.29(1)$ & $-1.28(1)$ & $-1.29(1)$ & $-1.30(1)$ & $-1.30(1)$ & - & $-1.29(1)$ \\
\hline $\mathrm{rms}^{\mathrm{c}} / \mathrm{kHz}$ & 0.7 & 1.8 & 0.8 & 0.9 & 0.9 & 1.0 & 0.9 & 0.9 & 0.4 & 0.9 \\
\hline \# lines & 244 & 18 & 66 & 63 & 63 & 62 & 63 & 62 & 30 & 41 \\
\hline
\end{tabular}

${ }^{a}$ Spectroscopic constants from reference 17: $\mathrm{A}=5202.103(46) \mathrm{MHz}, \mathrm{B}=972.68072(62) \mathrm{MHz}, \mathrm{C}=819.62733(61) \mathrm{MHz}, \Delta_{J}=0.0689(11) \mathrm{kHz}, \Delta_{J K}=-0.209(16)$ $\mathrm{kHz}, \Delta_{K}=[0.0], \delta_{J}=0.01210(77) \mathrm{kHz}, \delta_{K}=[0.0], \chi_{\mathrm{aa}}=2.701(12) \mathrm{MHz}, \chi_{\mathrm{bb}}=-1.444(15) \mathrm{MHz}, \chi_{\mathrm{cc}=}-1.258(15) \mathrm{MHz}$.

${ }^{\mathrm{b}} \mathrm{CD}$ constants in square brackets were fixed to the value from the parent.

${ }^{\mathrm{c}}$ microwave rms $=\sqrt{\frac{\sum(\text { obs }- \text { calc })^{2}}{\# \text { lines }}}$ 
Table 2. Ground State Spectroscopic Constants and Standard Errors Obtained for PhNCS and Its Isotopologues.

\begin{tabular}{|c|c|c|c|c|c|c|c|c|c|c|}
\hline & $\mathrm{PhNCS}$ & ${ }^{13} \mathrm{C} 1$ & ${ }^{13} \mathrm{C} 2$ & ${ }^{13} \mathrm{C} 3$ & ${ }^{13} \mathrm{C} 4$ & ${ }^{13} \mathrm{C} 5$ & ${ }^{13} \mathrm{C} 6$ & ${ }^{13} \mathrm{C} 7$ & ${ }^{15} \mathrm{~N}$ & ${ }^{34} \mathrm{~S}$ \\
\hline \multicolumn{11}{|c|}{ Rotational Constants $^{a} / \mathrm{MHz}$} \\
\hline$A$ & $5218.6948(5)$ & $5214.74(5)$ & $5158.17(5)$ & $5115.35(5)$ & $5209.78(7)$ & $5173.07(7)$ & $5131.96(6)$ & $5215.57(6)$ & $5196.16(3)$ & $5214.65(2)$ \\
\hline$B$ & $634.47256(3)$ & $634.28085(6)$ & $633.86807(8)$ & $630.53423(7)$ & $626.39418(8)$ & $628.17206(8)$ & $632.74748(7)$ & $631.50203(7)$ & $633.97616(4)$ & $616.23303(3)$ \\
\hline$C$ & $565.63308(3)$ & $565.43467(7)$ & $564.43402(9)$ & $561.27790(8)$ & $559.1056(1)$ & $560.0868(1)$ & $563.22739(9)$ & $563.23546(9)$ & $564.97452(5)$ & $551.04506(4)$ \\
\hline \multicolumn{11}{|c|}{ Centrifugal Distortion Constants ${ }^{b} / \mathrm{kHz}$} \\
\hline$\Delta_{J}$ & $0.04812(5)$ & $0.0478(7)$ & $0.0494(9)$ & $0.0475(8)$ & $0.046(1)$ & $0.049(1)$ & $0.0483(9)$ & $0.048(1)$ & $0.0478(3)$ & $0.0463(2)$ \\
\hline$\Delta_{J K}$ & $-0.1303(8)$ & {$[-0.1303]$} & {$[-0.1303]$} & {$[-0.1303]$} & {$[-0.1303]$} & {$[-0.1303]$} & {$[-0.1303]$} & {$[-0.1303]$} & {$[-0.1303]$} & {$[-0.1303]$} \\
\hline$\Delta_{K}$ & 7.1(1) & [7.1] & [7.1] & [7.1] & [7.1] & [7.1] & [7.1] & [7.1] & [7.1] & [7.1] \\
\hline$\delta_{J}$ & $0.00840(3)$ & {$[0.00840]$} & {$[0.00840]$} & {$[0.00840]$} & {$[0.00840]$} & {$[0.00840]$} & {$[0.00840]$} & {$[0.00840]$} & {$[0.00840]$} & {$[0.00840]$} \\
\hline$\delta_{K}$ & $0.62(1)$ & {$[0.62]$} & {$[0.62]$} & {$[0.62]$} & {$[0.62]$} & {$[0.62]$} & {$[0.62]$} & {$[0.62]$} & {$[0.62]$} & {$[0.62]$} \\
\hline \multicolumn{11}{|c|}{${ }^{14} \mathrm{~N}$ Quadrupole Coupling Constants /MHz } \\
\hline$\chi_{\mathrm{aa}}$ & $1.935(1)$ & $1.94(1)$ & 1.93(1) & $1.93(1)$ & $1.95(1)$ & $1.93(1)$ & $1.93(1)$ & $1.94(1)$ & - & $1.95(1)$ \\
\hline$\chi_{\mathrm{bb}}$ & $-1.452(1)$ & $-1.43(2)$ & $-1.45(2)$ & $-1.43(2)$ & $-1.41(2)$ & $-1.40(2)$ & $-1.43(2)$ & $-1.41(2)$ & - & $-1.49(2)$ \\
\hline$\chi_{c c}$ & $-0.483(1)$ & $-0.51(2)$ & $-0.49(2)$ & $-0.51(2)$ & $-0.53(2)$ & $-0.52(2)$ & $-0.51(2)$ & $-0.53(2)$ & - & $-0.45(2)$ \\
\hline $\mathrm{rms}^{\mathrm{c}} / \mathrm{kHz}$ & 0.8 & 0.7 & 0.9 & 0.8 & 1.0 & 1.0 & 0.9 & 0.9 & 0.7 & 1.0 \\
\hline \# lines & 214 & 30 & 27 & 30 & 30 & 29 & 29 & 27 & 20 & 65 \\
\hline
\end{tabular}

${ }^{a}$ Spectroscopic constants from reference 17: $\mathrm{A}=5219.03(38) \mathrm{MHz}, \mathrm{B}=634.4716(16) \mathrm{MHz}, \mathrm{C}=565.6340(17) \mathrm{MHz}, \Delta_{J}=0.0489(52) \mathrm{kHz}, \Delta_{J K}=-0.134(53) \mathrm{kHz}$, $\Delta_{K}=[0.0], \delta_{J}=0.00918(54) \mathrm{kHz}, \delta_{K}=[0.0], \chi_{\mathrm{aa}}=1.925(14) \mathrm{MHz}, \chi_{\mathrm{bb}}=-1.434(18) \mathrm{MHz}, \chi_{\mathrm{cc}=}-0.491(18) \mathrm{MHz}$.

${ }^{\mathrm{b}} \mathrm{CD}$ constants in square brackets were fixed to the value from the parent.

${ }^{c}$ microwave rms $=\sqrt{\frac{\sum(\text { obs }- \text { calc })^{2}}{\# \text { lines }}}$ 
Table 3. Mass Dependence $\left(\mathrm{r}_{\mathrm{m}}{ }^{(1)}\right)$ and Equilibrium $\left(\mathrm{r}_{\mathrm{e}}\right)$ (MP2/aug-cc-pVTZ) Structural Parameters (Bond Lengths in $\AA$, Angles in degrees) Determined for Phenyl Isocyanate and Phenyl Isothiocyanate.

\begin{tabular}{|c|c|c|c|c|}
\hline & \multicolumn{2}{|c|}{$\mathrm{PhNCO}$} & \multicolumn{2}{|c|}{$\mathrm{PhNCS}$} \\
\hline & $\mathrm{r}_{\mathrm{m}}^{(1)}$ & $\mathrm{r}_{\mathrm{e}}$ & $\mathrm{r}_{\mathrm{m}}^{(1)}$ & $\mathrm{r}_{\mathrm{e}}$ \\
\hline $\mathrm{r}(\mathrm{C} 1 \mathrm{C} 2)$ & $1.393(4)$ & 1.398 & $1.398(2)$ & 1.399 \\
\hline $\mathrm{r}(\mathrm{C} 2 \mathrm{C} 3)$ & $1.391(5)$ & 1.392 & $1.401(5)$ & 1.391 \\
\hline $\mathrm{r}(\mathrm{C} 3 \mathrm{C} 4)$ & $1.397(2)$ & 1.394 & $1.399(5)$ & 1.395 \\
\hline $\mathrm{r}(\mathrm{C} 4 \mathrm{C} 5)$ & $1.393(3)$ & 1.394 & $1.397(7)$ & 1.394 \\
\hline $\mathrm{r}(\mathrm{C} 5 \mathrm{C} 6)$ & $1.396(2)$ & 1.392 & $1.398(4)$ & 1.391 \\
\hline $\mathrm{r}(\mathrm{C} 1 \mathrm{C} 6)$ & $1.401(5)$ & 1.395 & $1.399(5)$ & 1.396 \\
\hline $\mathrm{r}(\mathrm{NC} 1)$ & $1.393(7)$ & 1.399 & $1.380(5)$ & 1.385 \\
\hline $\mathrm{r}(\mathrm{C} 7 \mathrm{~N})$ & $1.207(4)$ & 1.214 & $1.195(7)$ & 1.206 \\
\hline $\mathrm{r}(\mathrm{C} 7 \mathrm{O} / \mathrm{S})$ & $1.173(3)$ & 1.178 & $1.581(5)$ & 1.576 \\
\hline$\angle(\mathrm{C} 3 \mathrm{C} 2 \mathrm{C} 1)$ & $119.9(4)$ & 119.6 & $119.0(3)$ & 119.3 \\
\hline$\angle(\mathrm{C} 4 \mathrm{C} 3 \mathrm{C} 2)$ & $120.4(5)$ & 120.4 & $120.5(3)$ & 120.4 \\
\hline$\angle(\mathrm{C} 5 \mathrm{C} 4 \mathrm{C} 3)$ & $119.6(1)$ & 119.7 & $119.8(2)$ & 119.8 \\
\hline$\angle(\mathrm{C} 6 \mathrm{C} 5 \mathrm{C} 4)$ & $120.5(1)$ & 120.4 & $120.4(3)$ & 120.4 \\
\hline$\angle(\mathrm{C} 1 \mathrm{C} 6 \mathrm{C} 5)$ & $119.7(3)$ & 119.6 & $119.2(3)$ & 119.4 \\
\hline$\angle(\mathrm{C} 2 \mathrm{C} 1 \mathrm{C} 6)$ & $119.9(5)$ & 120.3 & $121.1(3)$ & 120.7 \\
\hline$\angle(\mathrm{NC} 1 \mathrm{C} 2)$ & $122.4(4)$ & 121.7 & $120.4(1)$ & 120.3 \\
\hline$\angle(\mathrm{C} 7 \mathrm{NC} 1)$ & $135.2(4)$ & 136.5 & $145.1(2)$ & 146.6 \\
\hline$\angle(\mathrm{NC} 7 \mathrm{O} / \mathrm{S})$ & $173.8(6)$ & 172.8 & $176.6(6)$ & 175.3 \\
\hline $\mathrm{c}_{\alpha}\left(\mathrm{u}^{1 / 2} \AA\right)$ & $0.0168(4)$ & & $-0.0123(8)$ & \\
\hline$\sigma\left(\mathrm{u} \AA^{2}\right)$ & 0.0055 & & 0.012 & \\
\hline
\end{tabular}


Table 4. Comparison between Experimental and Calculated (MP2 and B3LYP-D3BJ, aug-cc-pVTZ) Spectroscopic Constants for PhNCO and PhNCS. The Centrifugal Distortion Constants are Reported for Both Harmonic (H) and Anharmonic (A) Frequencies.

\begin{tabular}{|c|c|c|c|c|c|c|c|c|c|c|c|c|}
\hline \multirow[b]{2}{*}{ PhNCO } & \multicolumn{3}{|c|}{ Rotational Constants/MHz } & \multicolumn{3}{|c|}{${ }^{14} \mathrm{~N}$ Quadrupole Coupling Constants /MHz } & \multicolumn{6}{|c|}{ Centrifugal Distortion Constants $/ \mathrm{kHz}$} \\
\hline & $\boldsymbol{A}$ & B & $C$ & $\chi_{\mathrm{aa}}$ & $\chi_{b b}$ & $\chi_{\mathrm{cc}}$ & & $\Delta_{J}$ & $\Delta_{J K}$ & $\Delta_{K}$ & $\delta_{J}$ & $\delta_{K}$ \\
\hline Experimental & $5201.7138(3)$ & $972.68164(2)$ & $819.62560(2)$ & $2.7241(4)$ & $-1.4474(4)$ & $-1.2766(4)$ & & $0.07264(9)$ & $-0.2403(5)$ & $3.39(6)$ & $0.01669(7)$ & $0.451(7)$ \\
\hline \multirow[t]{2}{*}{ B3LYP-D3BJ } & 5324.92 & 960.94 & 813.66 & 2.768 & -1.6178 & -1.1502 & $\mathbf{H}$ & 0.0403 & 2.5229 & 1.096 & 0.0029 & 1.0595 \\
\hline & & & & & & & $\mathbf{A}$ & 0.0630 & -0.0993 & 3.1301 & 0.0142 & 0.4568 \\
\hline \multirow[t]{2}{*}{ MP2 } & 5244.99 & 964.85 & 814.94 & 2.7296 & -1.2398 & -1.4898 & $\mathbf{H}$ & 0.0451 & 2.3226 & 0.3723 & 0.0043 & 1.0225 \\
\hline & \multicolumn{3}{|c|}{ Rotational Constants/MHz } & \multicolumn{3}{|c|}{${ }^{14} \mathrm{~N}$ Quadrupole Coupling Constants /MHz } & \multicolumn{6}{|c|}{ Centrifugal Distortion Constants $/ \mathrm{kHz}$} \\
\hline$P h N C S$ & $\boldsymbol{A}$ & B & C & $\chi_{\text {aa }}$ & $\chi_{b b}$ & $\chi_{c c}$ & & $\Delta_{J}$ & $\Delta_{J K}$ & $\Delta_{K}$ & $\delta_{J}$ & $\delta_{K}$ \\
\hline Experimental & $5218.6948(5)$ & $634.47256(3)$ & $565.63308(3)$ & $1.935(1)$ & $-1.452(1)$ & $-0.483(1)$ & & $0.04812(5)$ & $-0.1303(8)$ & $7.1(1)$ & $0.00840(3)$ & $0.62(1)$ \\
\hline \multirow[t]{2}{*}{ B3LYP-D3BJ } & 5358.97 & 625.77 & 560.34 & 1.8987 & -1.5235 & -0.3752 & $\mathbf{H}$ & 0.0188 & 5.4177 & 57.3063 & 0.0040 & -1.6672 \\
\hline & & & & & & & $\mathbf{A}$ & 0.0407 & 0.1191 & 6.2556 & 0.0070 & 0.6331 \\
\hline MP2 & 5283.70 & 627.90 & 561.21 & 1.9689 & -1.1443 & -0.8246 & $\mathbf{H}$ & 0.0181 & 5.1064 & 58.0997 & 0.0041 & -1.5284 \\
\hline
\end{tabular}


Table 5. The ${ }^{14} \mathrm{~N}$ Quadrupole Coupling Constants (in MHz) in the Quadrupole Tensor Frame (x, $\mathrm{y}, \mathrm{z}$ ) and Results from the Townes-Dailey Analysis of PhNCO and PhNCS.

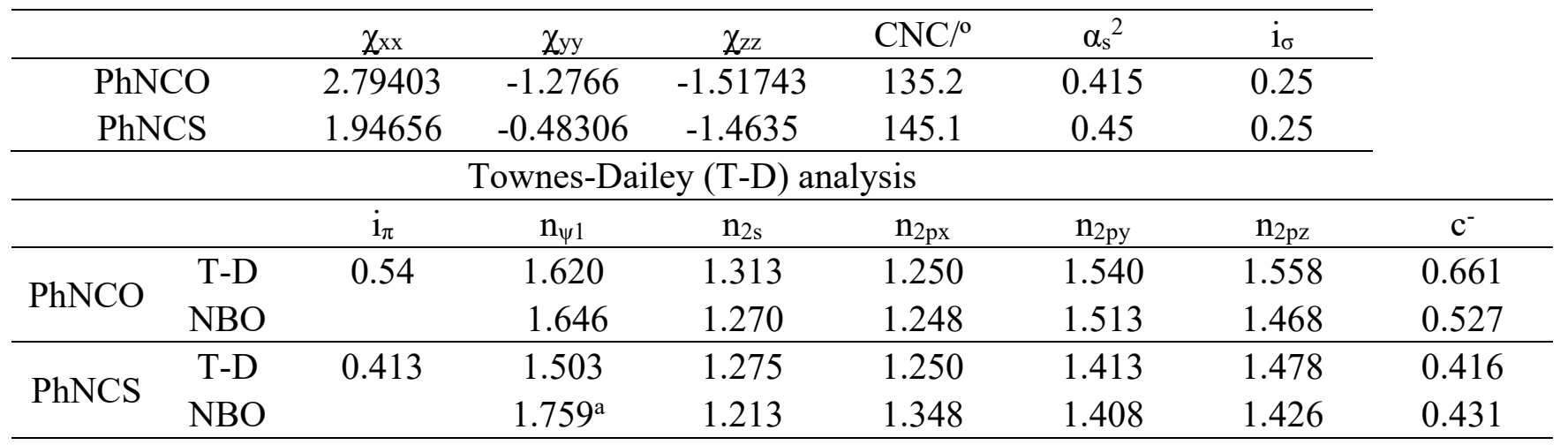

${ }^{\mathrm{a}}$ The NBO results do not identify a lone pair on nitrogen but rather predict a second $\pi$ bond with $\mathrm{C} 7$ as described in the text. This value is the occupancy of that orbital that approximates the lone pair. 


\section{References}

(1) Snyder, L. E.; Buhl, D. Interstellar Isocyanic Acid. Astrophys. J. 1972, 177, 619.

(2) Frerking, M. A.; Linke, R. A.; Thaddeus, P. Interstellar Isothiocyanic Acid. Astrophys. J. 1979, 234, L143.

(3) Halfen, D. T.; Ilyushin, V. V; Ziurys, L. M. Interstellar Dectection of Methyl Isothiocyanate $\mathrm{CH}_{3} \mathrm{NCO}$ in Sgr B2(N): A Link from Molecular Clouds to Comets. Astrophys. J. Lett. 2015, 812 (1), L5.

(4) Marcelino, N.; Agúndez, M.; Cernicharo, J.; Roueff, E.; Tafalla, M. Discovery of the Elusive Radical $\mathrm{NCO}$ and Confirmation of $\mathrm{H}_{2} \mathrm{NCO}^{+}$in Space. Astron. Astrophys. 2018, 612, L10.

(5) Hocking, W. H.; Gerry, M. C. L.; Winnewisser, G. The Microwave and Millimetre Wave Spectrum, Molecular Constants, Dipole Moment, and Structure of Isocyanic Acid, HNCO. Can. J. Phys. 1975, 53 (19), 1869-1901.

(6) Beard, C. I.; Dailey, B. P. The Structure and Dipole Moment of Isothiocyanic Acid. J. Chem. Phys. 1950, 18 (11), 1437-1441.

(7) Lett, R. G.; Flygare, W. H. Microwave Spectrum, Barrier to Internal Rotation, ${ }^{14}$ N Nuclear Quadrupole Interaction, and Normal-Coordinate Analysis in Methylisocyanate, Methylisothiocyanate, and Methylthiocyanate. J. Chem. Phys. 1967, 47 (11), 4730-4750.

(8) Ross, S. C.; Cooper, T. A.; Firth, S.; Kroto, H. W.; Walton, D. R. M. The Microwave Spectrum and Semirigid Bender Analysis of Isocyanatoethyne, HCCNCO. J. Mol. Spectrosc. 1992, 152, 152-167.

(9) Sun, W.; Davis, R. L.; Thorwirth, S.; Harding, M. E.; Van Wijngaarden, J. A Highly Flexible Molecule: The Peculiar Case of Ethynyl Isothiocyanate HCCNCS. J. Chem. Phys. 
2018, $149(10) 104304$.

(10) Hocking, W. H.; Gerry, M. C. L. The Microwave Spectrum of Cyanogen Isocyanate (NCNCO). J. Mol. Spectrosc. 1976, 59 (3), 338-354.

(11) King, M. A.; Kroto, H. W.; Landsberg, B. M. Microwave Spectrum of the Quasilinear Molecule, Cyanogen Isothiocyanate (NCNCS). J. Mol. Spectrosc. 1985, 113 (1), 1-20.

(12) Kirby, C.; Kroto, H. W. Microwave and Photoelectron Study of Cis- and Trans-Isocyanato Ethene, $\mathrm{CH}_{2} \mathrm{CHNCO}$ (Vinyl Isocyanate). J. Mol. Spectrosc. 1978, 70, 216-228.

(13) Caminati, W. The Microwave Spectrum of S-Trans Vinyl Isothiocyanate. J. Mol. Struct. 1988, 190, 227-233.

(14) Higgins, R. J.; Combs, L. L.; Malloy, T. B.; Cook, R. L. Low Resolution Microwave Spectra and Indo Calculations of Phenylisothiocyanate and Phenylisocyanate. J. Mol. Struct. 1975, $28(1), 121-127$.

(15) Bouchy, A.; Roussy, G. Microwave Spectrum of Phenyl Isocyanate. J. Mol. Spectrosc. 1977, 65 (3), 395-404.

(16) Onda, M.; Kambayashi, S.; Sakaizumi, T.; Yamaguchi, I. Microwave Spectrum of Phenylisothiocyanate. J. Mol. Struct. 1976, 34 (2), 299-302.

(17) Kasten, W.; Dreizler, H. Nitrogen Quadrupole Coupling in the Microwave Spectra of Phenyl Isocyanate and Phenyl Isothiocyanate. Z. Naturforsch. A 1987, 42 (1), 79-82.

(18) Stephenson, C. .; Coburn, W. .; Wilcox, W. . The Vibrational Spectra and Assignments of Nitrobenzene, Phenyl Isocyanate, Phenyl Isothiocyanate, Thionylaniline and Anisole. Spectrochim. Acta 1961, 17 (9-10), 933-946.

(19) Mani, P.; Umamaheswari, H.; Dominic Joshua, B.; Sundaraganesan, N. Molecular Structure, Vibrational Spectra and NBO Analysis of Phenylisothiocyanate by Density 
Functional Method. J. Mol. Struct. THEOCHEM 2008, 863 (1-3), 44-49.

(20) Foerner, W.; Badawi, H. M. Rotational Barriers and Vibrational Spectra of Phenyl Ketene, Azide, and Isocyanate. J. Theor. Comput. Chem. 2010, 09 (02), 511-529.

(21) Brown, G. G.; Dian, B. C.; Douglass, K. O.; Geyer, S. M.; Pate, B. H. The Rotational Spectrum of Epifluorohydrin Measured by Chirped-Pulse Fourier Transform Microwave Spectroscopy. J. Mol. Spectrosc. 2006, 238 (2), 200-212.

(22) Balle, T. J.; Flygare, W. H. Fabry-Perot Cavity Pulsed Fourier Transform Microwave Spectrometer with a Pulsed Nozzle Particle Source. Rev. Sci. Instrum. 1981, 52 (1), 33-45.

(23) Sedo, G.; Van Wijngaarden, J. Fourier Transform Microwave Spectra of a New Isomer of OCS- $\mathrm{CO}_{2}$. J. Chem. Phys. 2009, 131 (4), 044303.

(24) Evangelisti, L.; Sedo, G.; Van Wijngaarden, J. Rotational Spectrum of 1,1,1-Trifluoro-2Butanone Using Chirped-Pulse Fourier Transform Microwave Spectroscopy. J. Phys. Chem. A 2011, 115 (5), 685-690.

(25) Frisch, M. J.; Trucks, G. W.; Schlegel, H. B.; Scuseria, G. E.; Robb, M. A.; Cheeseman, J. R.; Scalmani, G.; Barone, V.; Petersson, G. A.; Nakatsuji, H.; et al. Gaussian 16, Revision B.01. Wallingford CT 2010.

(26) Sun, W.; Van Wijngaarden, J. Isothiocyanato-Containing Carbon Chains: The Laboratory Detection of HCCCCNCS and NCCCNCS via Rotational Spectroscopy. J. Phys. Chem. A 2018, 7659-7665.

(27) Glendening, E. D.; Badenhoop, J. K.; Reed, A. E; Carpenter, J. E.; Bohmann, J. A.; Morales, C. M.; Landis, C. R.; Weinhold, F. NBO 6.0 (Theoretical Chemistry Institute, University of Wisconsin, Madison, WI, 2013); http://nbo6.chem.wisc.edu/

(28) Pickett, H. M. The Fitting and Prediction of Vibration-Rotation Spectra with Spin 
Interactions. J. Mol. Spectrosc. 1991, 148 (2), 371-377.

(29) Watson, J. K. G. Determination of Centrifugal Distortion Coefficients of Asymmetric-Top Molecules. III. Sextic Coefficients. J. Chem. Phys. 1968, 48 (10), 4517-4524.

(30) Kraitchman, J. Determination of Molecular Structure from Microwave Spectroscopic Data. Am. J. Phys. 1953, 21 (1), 17-24.

(31) Watson, J. K. G.; Roytburg, A.; Ulrich, W. Least-Squares Mass-Dependence Molecular Structures. J. Mol. Spectrosc. 1999, 196 (1), 102-119.

(32) Desyatnyk, O.; Pszczółkowski, L.; Thorwirth, S.; Krygowski, T. M.; Kisiel, Z. The Rotational Spectra, Electric Dipole Moments and Molecular Structures of Anisole and Benzaldehyde. Phys. Chem. Chem. Phys. 2005, 7 (8), 1708-1715.

(33) Sun, W.; Lozada, I. B.; Van Wijngaarden, J. Fourier Transform Microwave Spectroscopic and $\mathrm{Ab}$ Initio Study of the Rotamers of 2-Fluorobenzaldehyde and 3-Fluorobenzaldehyde. J. Phys. Chem. A 2018, 122 (8), 2060-2068.

(34) Kisiel, Z. PROSPE http://www.ifpan.edu.pl/ kisiel/prospe.htm (accessed Dec 3, 2018).

(35) Kamaee, M.; Sun, M.; Luong, H.; Van Wijngaarden, J. Investigation of Structural Trends in Mono-, Di-, and Pentafluorobenzonitriles Using Fourier Transform Microwave Spectroscopy. J. Phys. Chem. A 2015, 119 (41), 10279-10292.

(36) Alonso, J. L.; Peña, I.; López, J. C.; Alonso, E.; Vaquero, V. The Shape of the Simplest Non-Proteinogenic Amino Acid $\alpha$-Aminoisobutyric (Aib). Chem. - A Eur. J. 2018, 1-8.

(37) Gordy, W.; Cooke, R. L. Microwave Molecular Spectra, 3rd edition; Wiley: New York, 1984.

(38) Van Dijk, C. W.; Sun, M.; Van Wijngaarden, J. Microwave Rotational Spectra and Structures of 2-Fluoropyridine and 3-Fluoropyridine. J. Phys. Chem. A 2012, 116 (16), 
4082-4088.

(39) Van Dijk, C. W.; Sun, M.; Van Wijngaarden, J. Investigation of Structural Trends in Difluoropyridine Rings Using Chirped-Pulse Fourier Transform Microwave Spectroscopy and Ab Initio Calculations. J. Mol. Spectrosc. 2012, 280 (1), 34-41.

(40) Novick, S. E. Extended Townes-Dailey Analysis of the Nuclear Quadrupole Coupling Tensor. J. Mol. Spectrosc. 2011, 267, 13-18.

(41) Chrostowska, A.; Darrigan, C.; Dargelos, A.; Graciaa, A.; Guillemin, J.-C.

Isoselenocyanates versus Isothiocyanates and Isocyanates. J. Phys. Chem. A 2018, 122

(11), 2894-2905. 
TOC Graphic

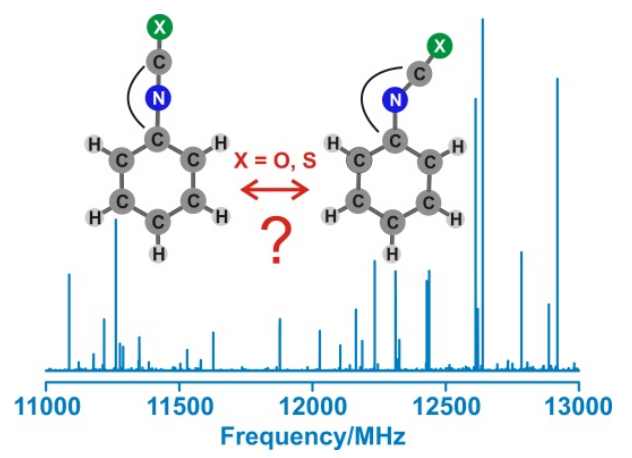

